\title{
Tetrahydroquinoline-Capped Histone Deacetylase 6 Inhibitor SW-101 Ameliorates Pathological Phenotypes in a Charcot-Marie-Tooth Type 2A Mouse Model
}

Sida Shen ${ }^{1, \dagger}$, Cristina Picci ${ }^{3, \dagger}$, Kseniya Ustinova ${ }^{4}$, Veronick Benoy ${ }^{5}, Z s o ́ f i a ~ K u t i l^{4}$, Guiping Zhang $^{1}$, Maurício T. Tavares ${ }^{1, \mathbb{1}, \text { Jiři Pavličé }}{ }^{4}$, Chad A. Zimprich ${ }^{6}$, Matthew B. Robers ${ }^{6}$, Ludo Van Den Bosch ${ }^{5}$, Cyril Bařinka*.4, Brett Langley ${ }^{* 3}$, and Alan P. Kozikowski ${ }^{* 2}$

${ }^{1}$ Department of Medicinal Chemistry and Pharmacognosy, College of Pharmacy, University of Illinois at Chicago, Chicago, Illinois 60612, United States.

${ }^{2}$ Bright Minds Biosciences, Toronto, ON M5H 3V9, Canada

${ }^{3}$ School of Health, The University of Waikato, Private Bag 3105, Hamilton 3240, New Zealand ${ }^{4}$ Institute of Biotechnology of the Czech Academy of Sciences, 25250 Vestec, Czech Republic. ${ }^{5}$ Laboratory of Neurobiology, Center for Brain \& Disease (VIB) and Leuven Brain Institute (LBI), KU Leuven, B-3000 Leuven, Belgium

${ }^{6}$ Promega Corporation, Madison, Wisconsin 53711, United States.

${ }^{\dagger}$ These authors contributed equally.

Corresponding authors:

*(C.B.) E-mail: Cyril.Barinka@ibt.cas.cz. Phone: +420-325-873-777.

*(B.L.) E-mail: blangley@,waikato.ac.nz. Phone: +64-7-838-4060.

*(A.P.K.) E-mail: alan@brightmindsbio.com. Phone: +1-773-793-5866. 
Table of Contents

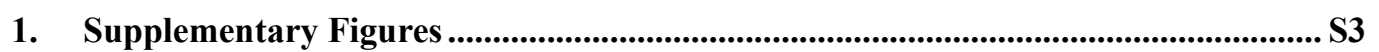

2. Supplementary Tables ........................................................................................................... S9

3. NMR Spectra and HPLC Purity Report for SW-100 and SW-101......................... S11 


\section{Supplementary Figures}

A

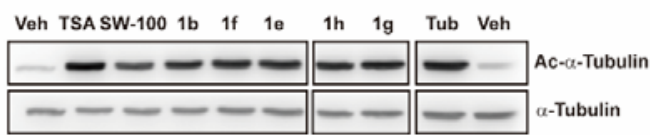

Ratio Ac- $\alpha$-tub/ $\alpha$-tub (1 $\mu \mathrm{M})$

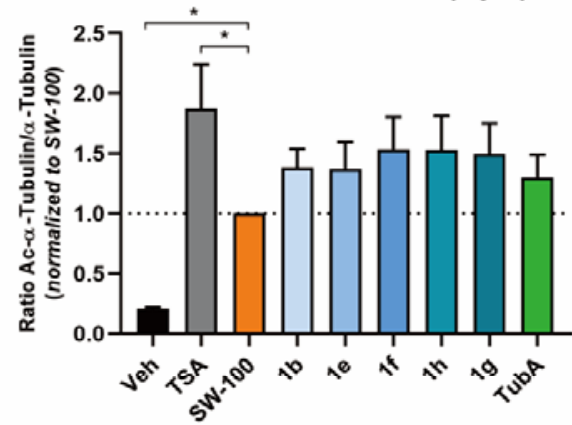

C

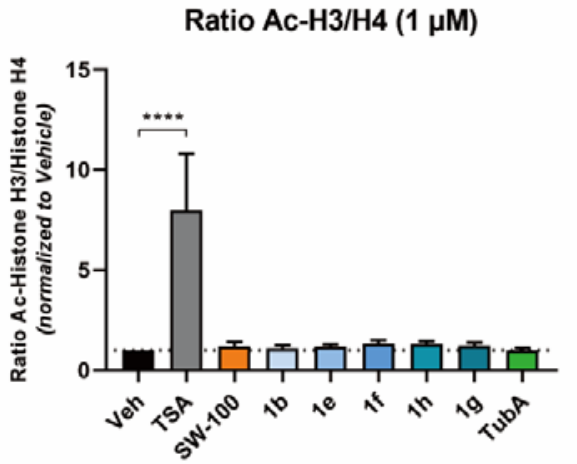

B

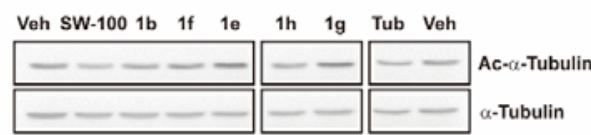

Ratio Ac- $\alpha$-tub/a-tub (0.01 $\mu \mathrm{M})$

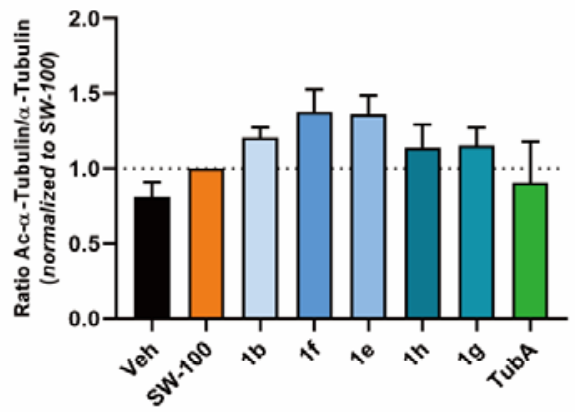

Figure S1. $\alpha$-Tubulin/histone acetylation evaluation of HDAC6 inhibitors $\mathbf{1 b}, \mathbf{1 e}, \mathbf{1 f}, \mathbf{1 g}$, and 1h in N2a cells. All compounds were tested at $1 \mu \mathrm{M}$ and $10 \mathrm{nM}$ concentrations, and acetylated $\alpha$-tubulin (Ac- $\alpha$-tub; A and B) and histone $3(\mathrm{Ac}-\mathrm{H} 3 ; \mathrm{C})$ were quantified by Western-Blot. Ratios of Ac- $\alpha$-tub to total $\alpha$-tubulin ( $\alpha$-tub) were normalized to SW-100. Ratios of Ac-H3and histone H4 (H4) were normalized to vehicle. TSA and TubA were tested as controls. Graphs represent means with SEM. Dunnett's multiple comparisons test. ${ }^{*} \mathrm{p}<0.05, * * * * \mathrm{p}<0.0001$. n $=3-6$. 

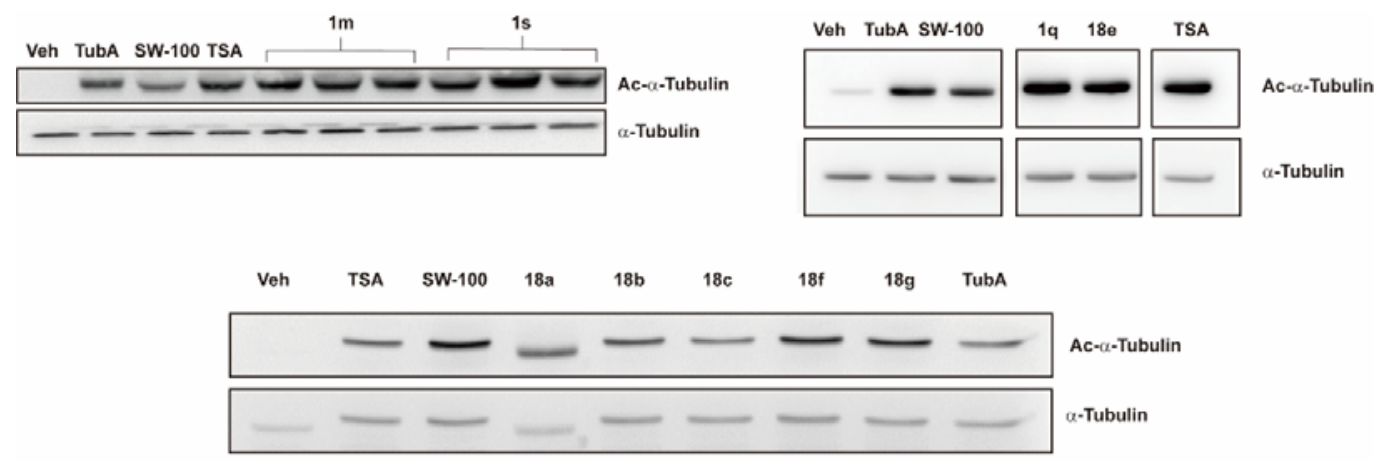

Ratio Ac- $\alpha-$ tub/ $\alpha-$ tub $(1 \mu \mathrm{M})$

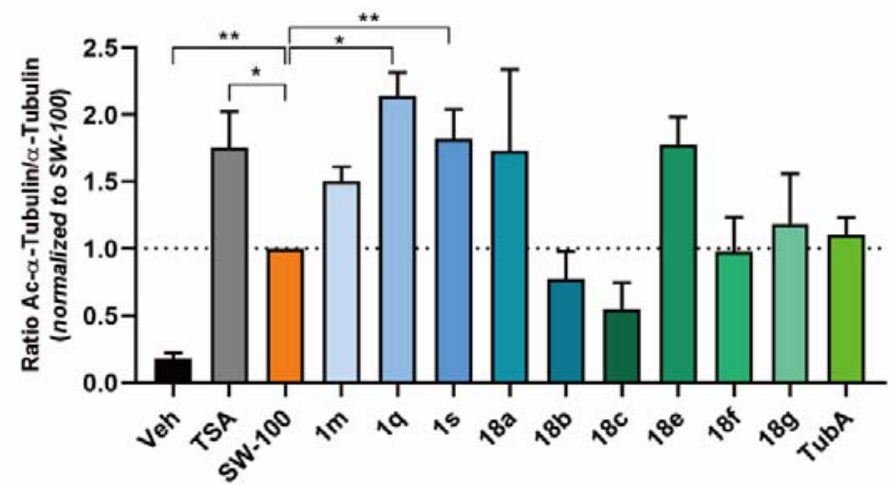

Figure S2. $\alpha$-Tubulin acetylation evaluation of HDAC6 inhibitors 1m, 1q, 1s, 18a-c, and 18e$\mathbf{g}$ in N2a cells at the concentration of $1 \mu \mathrm{M}$. Ac- $\alpha$-tub was quantified by Western-Blot. Ratios of Ac- $\alpha$-tub to total $\alpha$-tub were normalized to SW-100. TSA and TubA were tested as controls. Graphs represent means with SEM. Dunnett's multiple comparisons test. ${ }^{*} \mathrm{p}<0.05,{ }^{* *} \mathrm{p}<0.01$. $\mathrm{n}=3-13$. 


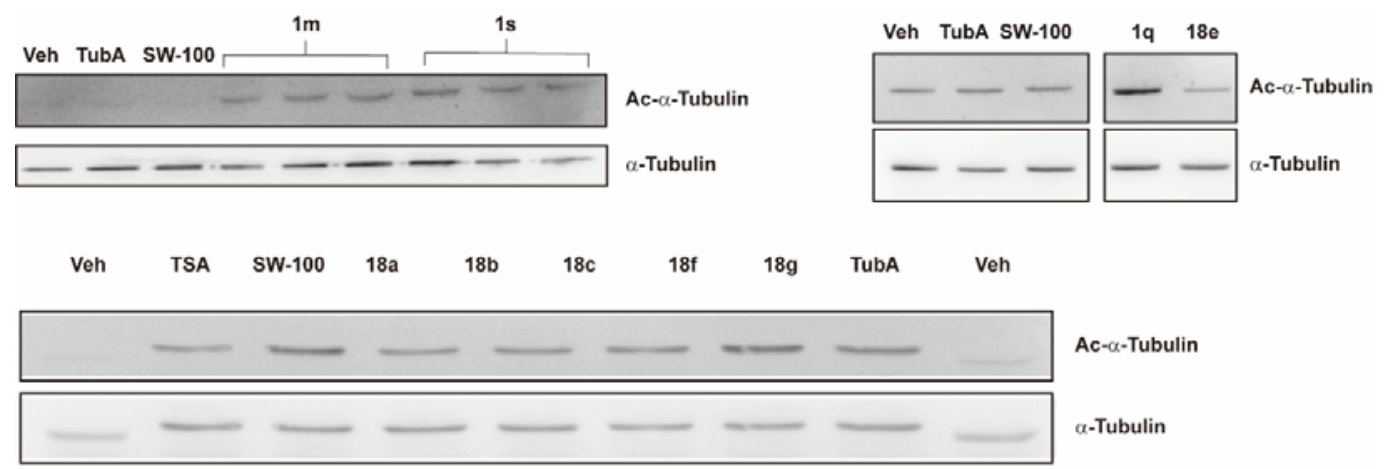

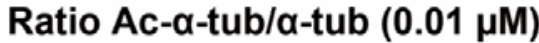

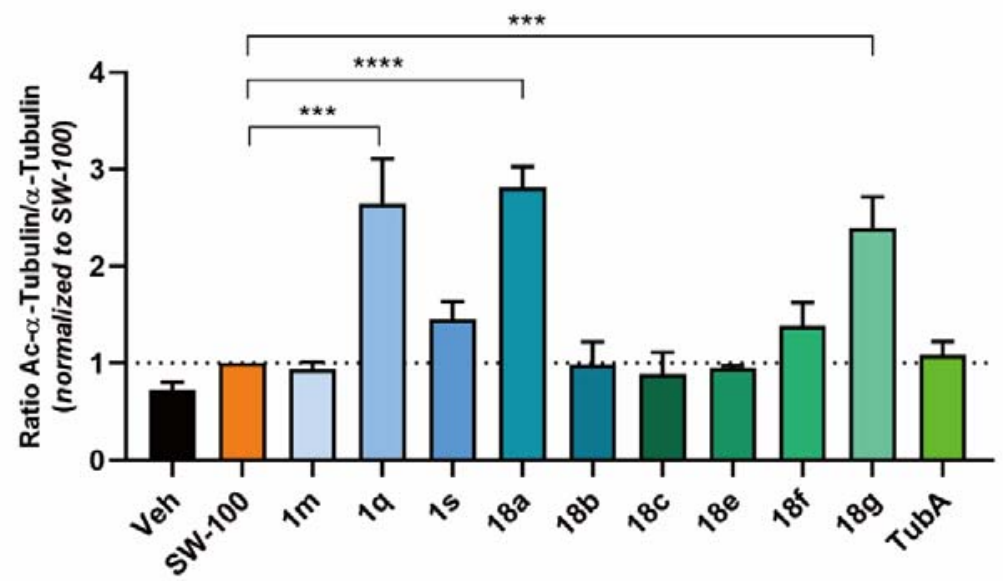

Figure S3. $\alpha$-Tubulin acetylation evaluation of HDAC6 inhibitors 1m, 1q, 1s, 18a-c, and 18e$\mathrm{g}$ in N2a cells at the concentration of $0.01 \mu \mathrm{M}$. Ac- $\alpha$-tub was quantified by Western-Blot. Ratios of Ac- $\alpha$-tub to total $\alpha$-tub were normalized to SW-100. TSA was tested as controls. Graphs represent means with SEM. Dunnett's multiple comparisons test. ***p $<0.001, * * * \mathrm{p}<0.0001$. $\mathrm{n}=3-11$. 

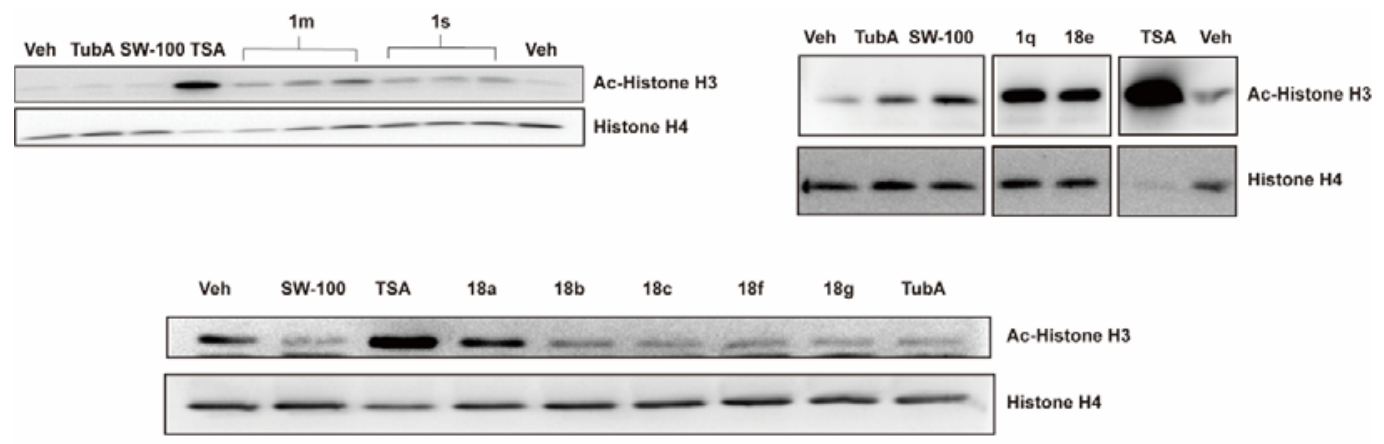

Ratio Ac-H3/H4 (1 $\mu \mathrm{M})$

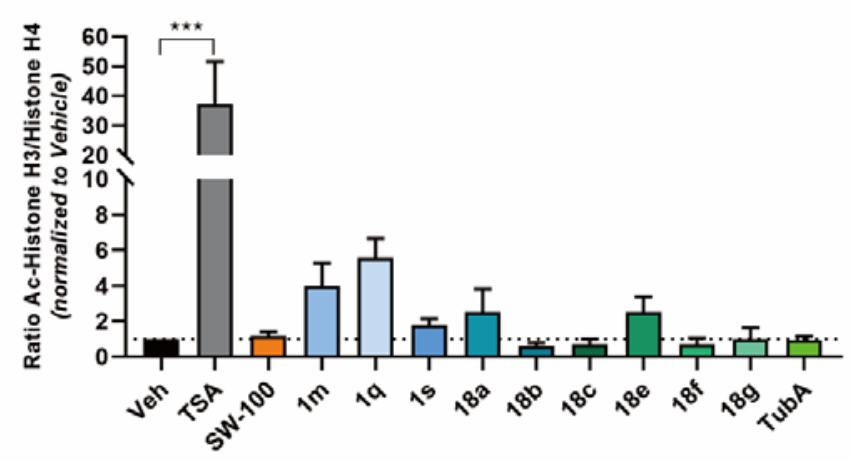

Figure S4. Histone acetylation evaluation of HDAC6 inhibitors 1m, 1q, 1s, 18a-c, and 18e-g in N2a cells at the concentration of $1 \mu \mathrm{M}$. Ac-H3 was quantified by Western-Blot. Ratios of Ac-H3and H4 were normalized to the vehicle group. TSA and TubA were tested as controls. Graphs represent means with SEM. Dunnett's multiple comparisons test. **p $<0.01 . \mathrm{n}=2-14$. 

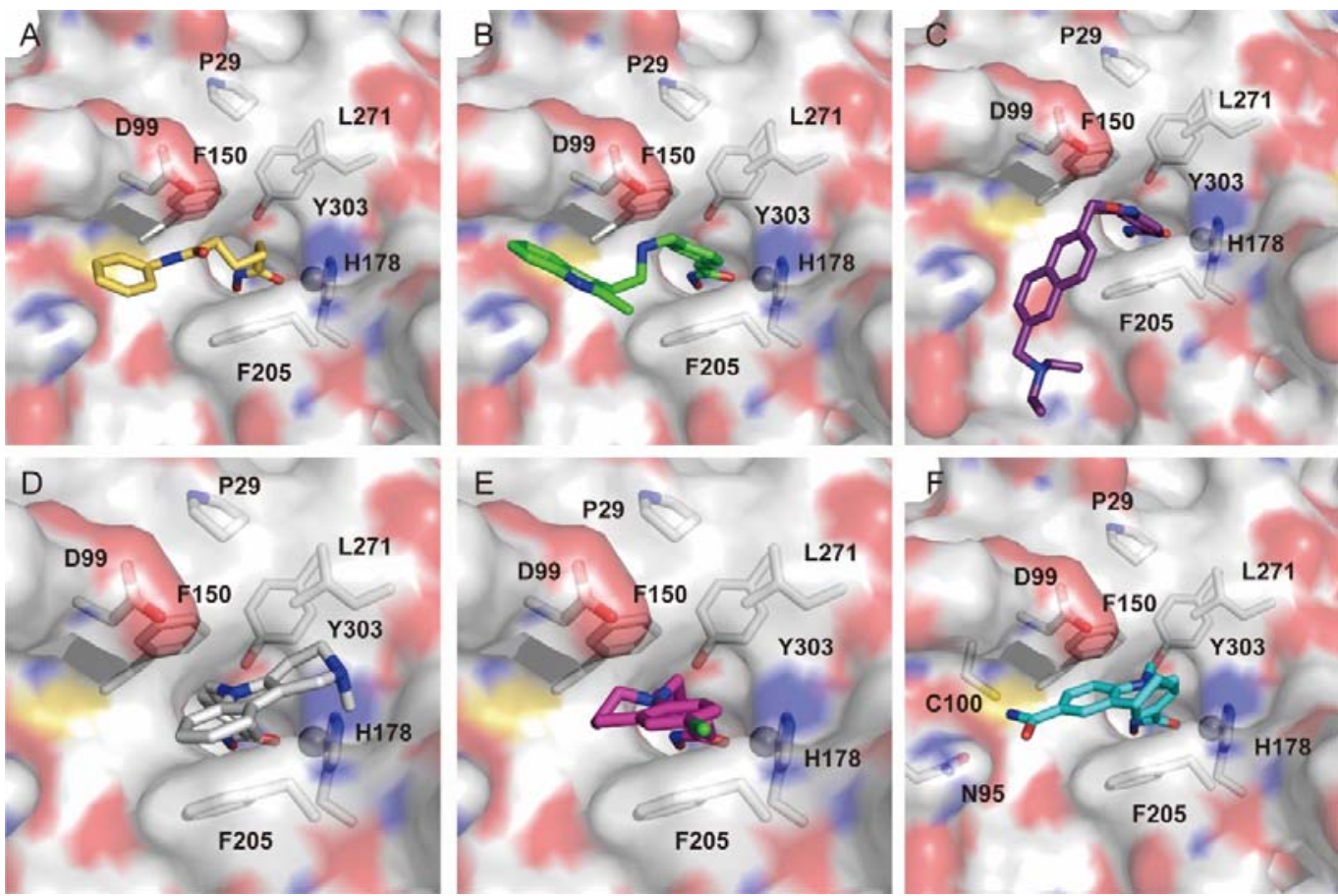

Figure S5. Molecular docking study results for Vorinostat (SAHA, A), Panobinostat (B), Givinostat (C), Tubastatin A (D), SW-100 (E), and SW-101 (F) based on the hHDAC1 crystal complex (PDB entry 5ICN).

A

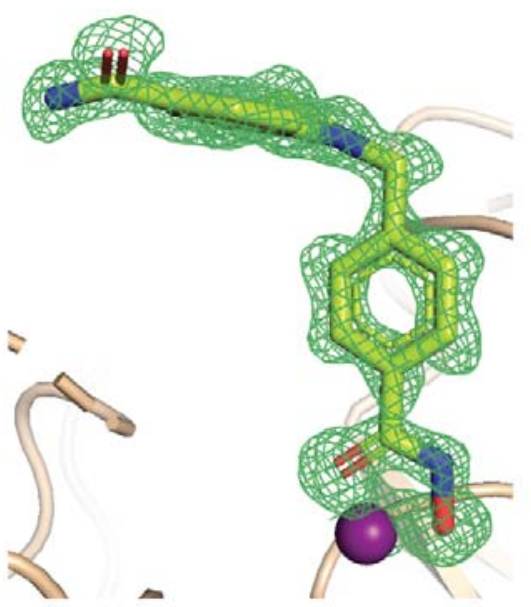

B

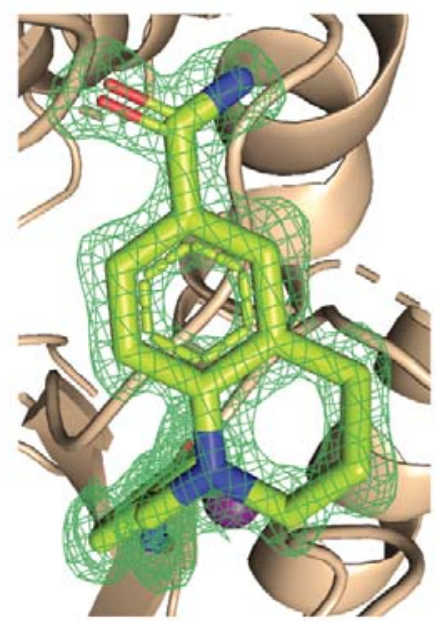

Figure S6. The omit $F O-F c$ difference map (green mesh) for zHDAC6-bound SW-101 (PDB entry $6 \mathrm{ZW} 1)$ is contoured at $4.0 \sigma$. The inhibitor is shown in stick representation with atoms colored lime green (carbon), red (oxygen), and blue (nitrogen). The active-site $\mathrm{Zn}^{2+}$ ion is shown as a purple sphere. 


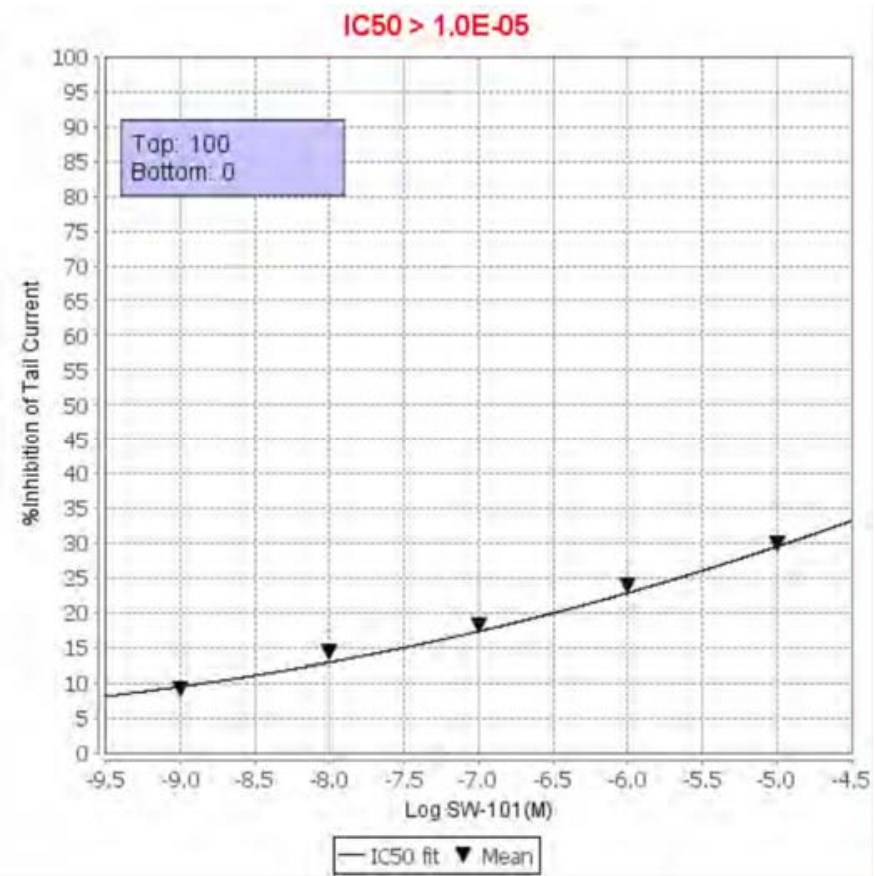

Figure S7. Inhibition of hERG potassium channel activity by SW-101 (1s) in CHOhERG cells by an automated patch-clamp electrophysiology assay. This assay was performed by AMRI Global, Albany, NY. 


\section{Supplementary Tables}

Table S1. Crystallization data collection and refinement statistics.

\begin{tabular}{|c|c|}
\hline Data collection & \\
\hline Inhibitor & SW-101 (1s) \\
\hline PDB code & 6ZW1 \\
\hline Space group & P21212 (18) \\
\hline Unit-cell parameters $a, b, c(\AA)$ & $83.93,94.16,51.49$ \\
\hline Wavelength $(\AA)$ & 0.9184 \\
\hline Resolution limits $(\AA)$ & $50-1.13(1.20-1.13)$ \\
\hline No of unique refl. & $152836(24308)$ \\
\hline Redundancy & $6.5(6.0)$ \\
\hline Completeness (\%) & $99.8(99.0)$ \\
\hline$I / \sigma(I)$ & $12.78(1.73)$ \\
\hline $\mathrm{R}_{\text {merge }}$ & $0.087(0.931)$ \\
\hline $\mathrm{CC} 1 / 2(\%)$ & $99.9(74.4)$ \\
\hline \multicolumn{2}{|l|}{ Refinement } \\
\hline Resolution limits $(\AA)$ & $47.08-1.13(1.16-1.13)$ \\
\hline Total number of reflections & $150734(10988)$ \\
\hline Number of reflections in working set & $148634(10837)$ \\
\hline Number of reflections in test set & $2100(151)$ \\
\hline $\mathrm{R} / \mathrm{R}_{\text {free }}(\%)$ & $13.79 / 15.94(25 / 23.7)$ \\
\hline Total number of non-H atoms & 3421 \\
\hline Number of non-H protein atoms & 2960 \\
\hline Number of inhibitor molecules & 1 \\
\hline Number of water molecules & 416 \\
\hline Average B-factor $\left(\AA^{2}\right)$ & 12.7850 \\
\hline Protein & 11.8952 \\
\hline Water molecules & 27.476 \\
\hline Inhibitor & 12.9742 \\
\hline \multicolumn{2}{|l|}{${ }^{\&}$ Ramachandran Plot (\%) } \\
\hline Most favored & 98 \\
\hline Additionally allowed & 2 \\
\hline Disallowed & 0 \\
\hline R.m.s. deviations: bond lengths $(\AA)$ & 0.011 \\
\hline bond angles $\left({ }^{\circ}\right)$ & 1.765 \\
\hline chiral centers $\left(\AA^{3}\right)$ & 0.100 \\
\hline
\end{tabular}

*Values in parentheses are for the highest resolution shells.

${ }^{\&}$ Structures were analyzed using the MolProbity package. 
Table S2. Mini-Ames genotoxicity assay results for SW-101 (1s) ${ }^{\mathrm{a}}$

\begin{tabular}{|c|c|c|c|c|c|c|c|c|}
\hline \multirow{3}{*}{$\begin{array}{c}\text { Test Conc. }(\boldsymbol{\mu M}) \\
\text { Rat liver S9 fraction } \\
\end{array}$} & \multicolumn{8}{|c|}{ Bacterial Strain (+/- S9), Positive Significance (- to +++$)$} \\
\hline & \multicolumn{2}{|c|}{ TA98 } & \multicolumn{2}{|c|}{ TA100 } & \multicolumn{2}{|c|}{ TA1535 } & \multicolumn{2}{|c|}{ TA1537 } \\
\hline & - & + & - & + & - & + & - & + \\
\hline 5 & - & - & - & - & - & - & - & - \\
\hline 10 & - & - & - & - & - & - & - & - \\
\hline 50 & - & - & - & - & - & - & - & - \\
\hline 100 & - & - & - & - & - & - & - & - \\
\hline 2-Aminoanthracene & - & +++ & - & ++ & - & ++ & - & +++ \\
\hline 9-Aminoacridine & - & - & - & - & - & - & +++ & +++ \\
\hline Quercetin & ++ & +++ & - & - & - & - & - & - \\
\hline Streptozotocin & - & - & +++ & +++ & +++ & +++ & - & - \\
\hline
\end{tabular}

${ }^{\mathrm{a}}$ Ames fluctuation assay was performed using four Salmonella strains, TA98, TA100, TA1535

and TA1537 by AMRI Global (https://www.amriglobal.com/, Albany, NY). TA98 and TA1537 detect frameshifts. TA100 detects base-pair substitutions leading to missense mutations. TA1535 detects base-pair substitutions and is used to identify certain mutagenic compounds which are not detected by TA98 and TA100. Metabolic activation was achieved by using rat liver S9 fraction. 
3. NMR Spectra and HPLC Purity Report for SW-100 (1a) and SW-101 (1s).

$\underline{\text { SW-100 }\left({ }^{1} \mathrm{H} \text { NMR, DMSO- } d_{6}\right)}$
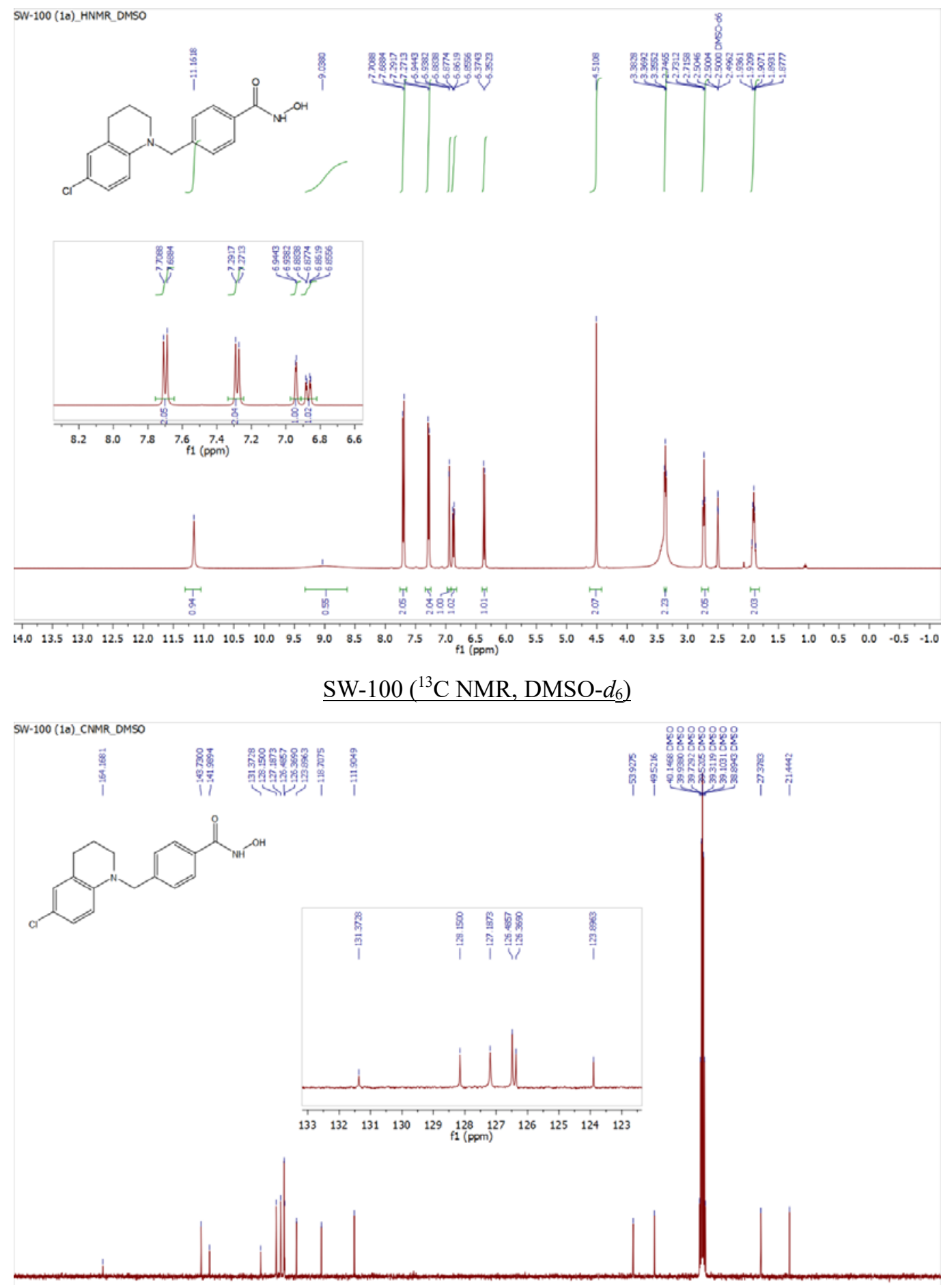

18

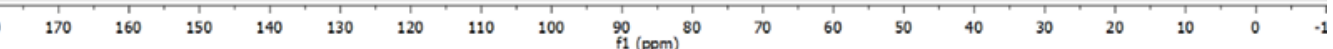




\section{$\underline{\text { SW-100 (HPLC, } 254 \mathrm{~nm} \mathrm{UV)}}$}

Data File C: \CHEM32\1\DATA \DEF_LC 2016-09-12 14-29-08\GPV-1-72-1.D Sample Name: GPV-1-72-1
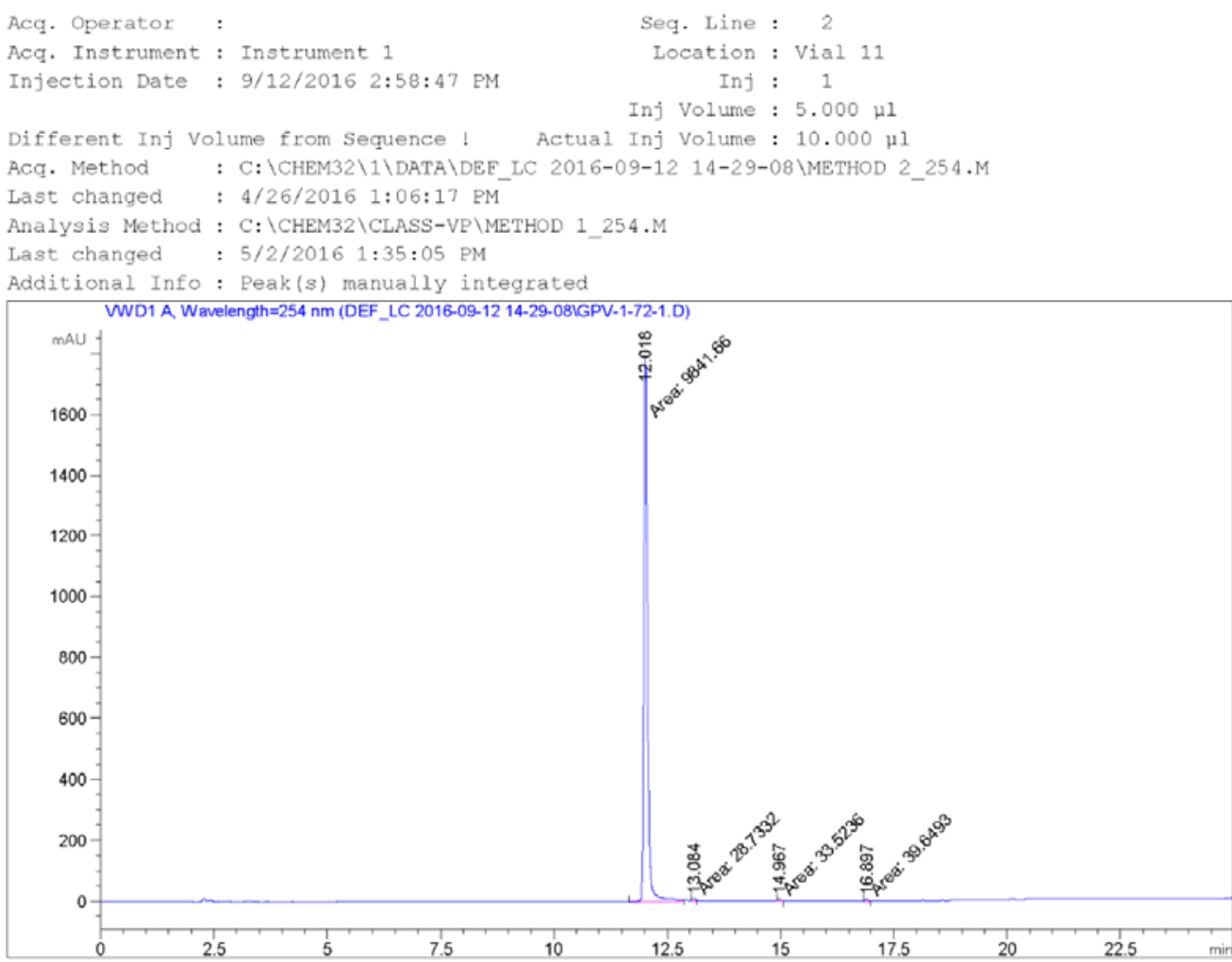

Area Percent Report

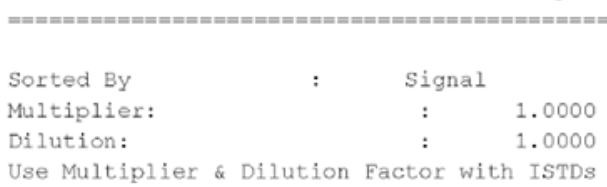

Signal 1: VWD1 $\mathrm{A}$, Wavelength $=254 \mathrm{~nm}$

\begin{tabular}{|c|c|c|c|c|c|c|}
\hline $\begin{array}{c}\text { eak } \\
\#\end{array}$ & $\begin{array}{l}\text { RetTime } \\
\text { [min] }\end{array}$ & Type & $\begin{array}{l}\text { Width } \\
\text { [min] }\end{array}$ & $\begin{array}{c}\text { Area } \\
{[\mathrm{mAU} * \mathrm{~s}]}\end{array}$ & $\begin{array}{l}\text { Height } \\
\text { [MAU] }\end{array}$ & $\begin{array}{c}\text { Area } \\
8\end{array}$ \\
\hline & & & & -- & - & +--- \\
\hline 1 & 12.018 & $\mathrm{MM}$ & 0.0916 & 9841.65625 & 1790.44043 & 98.9752 \\
\hline 2 & 13.084 & $\mathrm{MM}$ & 0.0764 & 28.73321 & 6.26936 & 0.2890 \\
\hline 3 & 14.967 & M & 0 & 33.52358 & 6.81858 & 0.3371 \\
\hline
\end{tabular}

Totals : $\quad 9943.56238 \quad 1809.42335$ 
Data File C: \CHEM32\1\DATA \DEF_LC 2016-09-12 14-29-08\GPV-1-72-1.D

Sample Name: GPV-1-72-1

$$
* * * \text { End of Report *** }
$$


$\underline{\text { SW-101 }\left({ }^{1} \mathrm{H} \text { NMR, DMSO- } d_{6}\right)}$

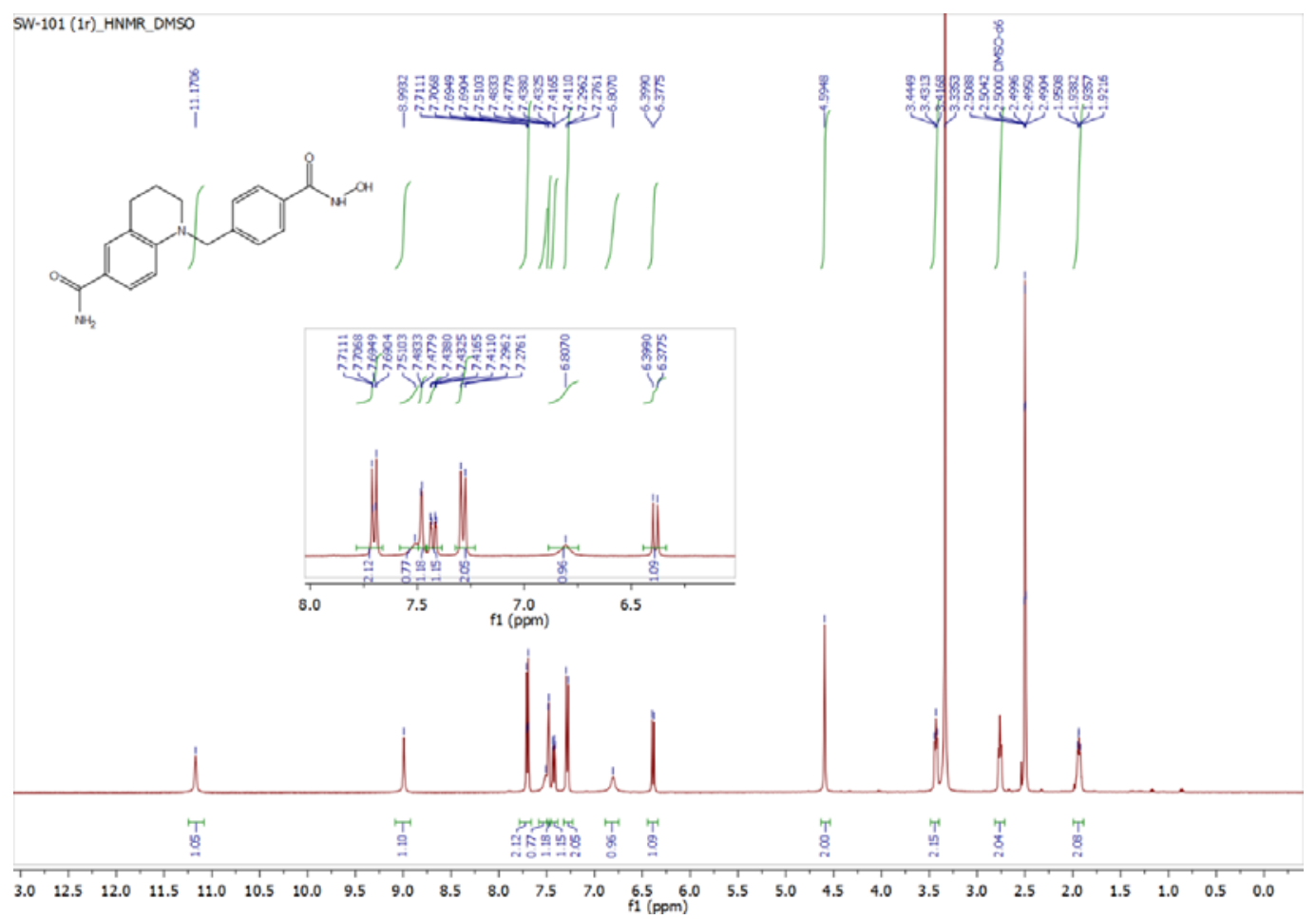

SW-101 $\left({ }^{13}\right.$ CNMR, DMSO- $\left.d_{6}\right)$

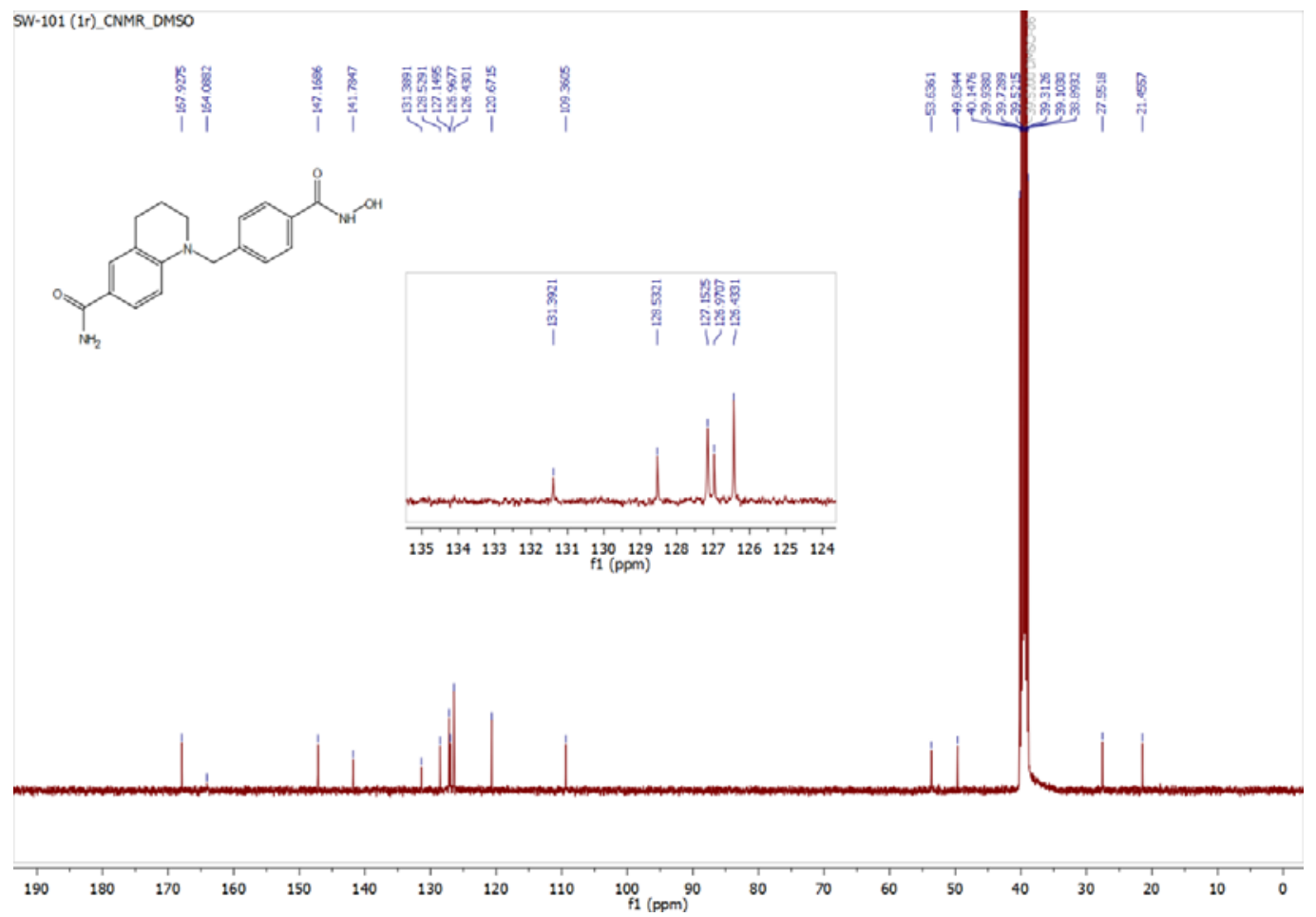




\section{$\underline{\text { SW-101 (HPLC, } 254 \mathrm{~nm} \mathrm{UV)}}$}

Data File C: \CHEM32\1\DATA \DEF_LC 2017-06-29 15-22-40\SS-4-100_0629.D Sample Name: SS-4-100_0629
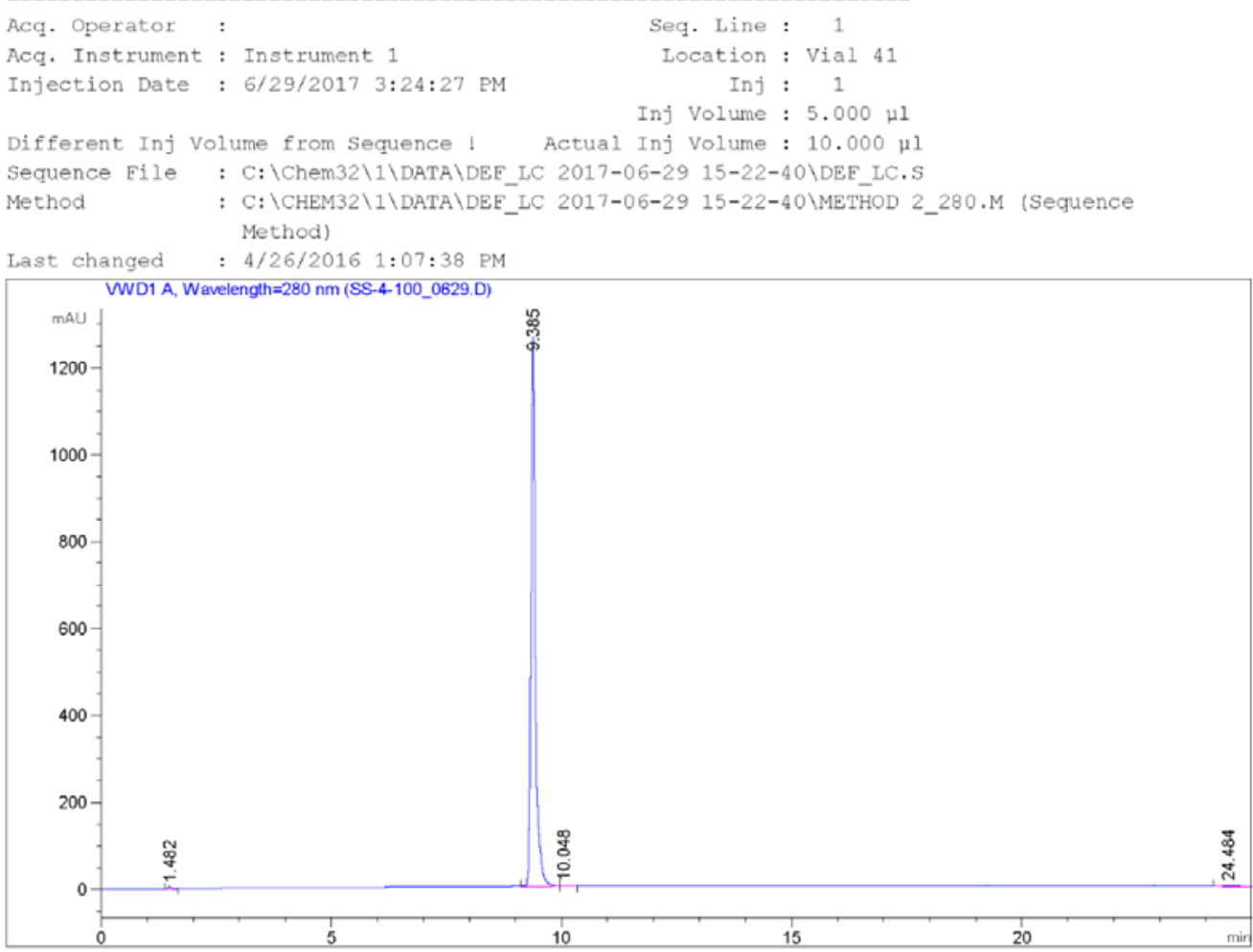

Area Percent Report

\begin{tabular}{|c|c|c|c|}
\hline Sorted By & : & Signal & \\
\hline Multiplier: & & : & 1.0000 \\
\hline Dilution: & & : & 1.0000 \\
\hline
\end{tabular}

Use Multiplier \& Dilution Factor with ISTDS

Signal 1: VWD1 $\mathrm{A}$, Wavelength=280 nm

\begin{tabular}{|c|c|c|c|c|c|c|}
\hline $\begin{array}{c}\text { Peak } \\
\#\end{array}$ & $\begin{array}{c}\text { RetTime } \\
\text { [min] }\end{array}$ & Type & $\begin{array}{l}\text { Width } \\
\text { [min] }\end{array}$ & $\begin{array}{c}\text { Area } \\
{[\mathrm{mAU} * \mathrm{~S}]}\end{array}$ & $\begin{array}{l}\text { Height } \\
\text { [mAU] }\end{array}$ & $\begin{array}{c}\text { Area } \\
8\end{array}$ \\
\hline- & |----- & & & - & --n--- & --n-- \\
\hline 1 & 1.482 & BV & 0.0869 & 35.96329 & 5.56368 & 0.4348 \\
\hline 2 & 9.385 & vV & 0.0935 & 8184.23242 & 1264.76526 & 98.9445 \\
\hline 3 & 10.048 & VB & 0.1120 & 15.57742 & 1.85429 & 0.1883 \\
\hline 4 & 24.484 & BBA & 0.2400 & 35.76236 & 1.75694 & 0.4324 \\
\hline
\end{tabular}

Totals : $\quad 8271.535491273 .94017$ 
Data File C: \CHEM32\1\DATA \DEF_LC 2017-06-29 15-22-40\SS-4-100_0629.D

Sample Name: Ss-4-100 0629

*** End of Report *** 